\title{
Efficacy of subantimicrobial-dose doxycycline against nitrosative stress in chronic periodontitis
}

\author{
Alina Elena PÂRVU ${ }^{1, *}$, Sandu Florin ALB ${ }^{2}$, Alexandra CRĂCIUN³ , Marian Aurel TAULESCU ${ }^{4}$ \\ ${ }^{1}$ Department of Pathophysiology, Faculty of Medicine, University of Medicine and Pharmacy "Iuliu Hațieganu," Cluj-Napoca 400012, \\ Romania; 'Department of Periodontology, Faculty of Medical Dentistry, University of Medicine and Pharmacy "Iuliu Hațieganum," \\ Cluj-Napoca 400012, Romania; ${ }^{3}$ Department of Biochemistry, Faculty of Medicine, University of Medicine and Pharmacy "Iuliu \\ Hațieganu," Cluj-Napoca 400012, Romania; ${ }^{4}$ Department of Pathology, Faculty of Veterinary Medicine, University of Agricultural and \\ Veterinary Sciences, Cluj-Napoca 400372, Romania
}

\begin{abstract}
Aim: To evaluate the effectiveness of subantimicrobial-dose doxycycline (SDD) as an adjunct to scaling and root planing (SRP) treatment against the nitrosative stress of moderate to advanced chronic periodontitis.

Methods: Adults with untreated chronic periodontitis $(n=174)$ were randomly administered SRP+SDD ( $n=87)(20$ mg of doxycycline twice daily) or SRP+placebo $(n=87)$ treatment for 3 months. At baseline and after 3 months, the probing depths (PD), bleeding on probing (BOP) and clinical attachment level (CAL) were measured, and a gingivomucosal biopsy was collected to assay the induction of nitric oxide synthase (iNOS) and 3-nitrotyrosine (3NT), and blood was collected to assay for total nitrites and nitrates ( $\mathrm{NO}_{\mathrm{x}}$ ) and $3 \mathrm{NT}$. Results: Compared to baseline, at the completion of treatment, significant decreases in the levels of tissue iNOS and 3NT and serum $\mathrm{NO}_{x}$ and $3 \mathrm{NT}$ were observed in both groups. SRP+SDD yielded a greater reduction in the gingivomucosal and serum nitrosative stress markers than did SRP+placebo. PD, BOP, and CAL reduction were correlated with the nitrosative stress parameters.

Conclusion: On a short-term basis, SDD therapy may be used as an adjunct to SRP treatment against nitrosative stress in moderate to advanced chronic periodontitis.
\end{abstract}

Keywords: nitric oxide; nitrosative stress; chronic periodontitis; scaling and root planing treatment; doxycycline; stomatology

Acta Pharmacologica Sinica (2013) 34: 247-254; doi: 10.1038/aps.2012.129; published online 12 Nov 2012

\section{Introduction}

Periodontitis is a multifactorial disease caused by the interaction of microbial agents present in the bacterial plaque, host susceptibility, and environmental factors ${ }^{[1]}$. When stimulated with bacteria or bacterial components, human monocytes, endothelial cells, macrophages, and gingival fibroblasts produce inflammatory mediators that perpetuate and amplify chronic periodontal inflammation through numerous autocrine and paracrine loops ${ }^{[2]}$. Epidemiological and interventional studies have revealed close associations between periodontal disease and systemic diseases, such as cardiovascular disease, preterm delivery and low birthweight, diabetes mellitus, respiratory infections, osteoporosis, and cancer ${ }^{[3-5]}$. The main causes of periodontitis-related systemic diseases are thought to be the oral-hematogenous spread of bacteria and the excessive release of periodontal inflammatory mediators ${ }^{[3]}$.

\footnotetext{
* To whom correspondence should be addressed. E-mail parvualinaelena@yahoo.com

Received 2012-04-09 Accepted 2012-08-06
}

Accordingly, therapeutic strategies for periodontal disease have been directed towards two different and complementary paths: antimicrobial therapy and host modulation. Three categories of host-modulating agents have been tested experimentally and clinically in periodontal therapy: antiproteinases (tetracyclines), anti-inflammatory drugs, and bone-sparing drugs (antiresorptive agents such as bisphosphonates) ${ }^{[2,4]}$. Tetracyclines are the most extensively investigated host-modulatory agents, and subantimicrobial-dose doxycycline (SDD) is widely used as an adjunct to scaling and root planing (SRP). The therapeutic effects of SDD are executed via collagenase, osteoclast and cytokine inhibition in alveolar bone, connective tissue and epithelium ${ }^{[6-8]}$.

In recent years, growing evidence of the involvement of nitric oxide (NO) and nitro-oxidative stress in chronic periodontitis has highlighted the need to improve diagnostic and treatment algorithms ${ }^{[9]}$. $\mathrm{NO}$ is produced in vivo from $L$-arginine by nitric oxide synthase (NOS). nNOS/NOS1 and eNOS/NOS3 are constitutive isoforms of the enzyme found in neuronal and endothelial cells, respectively. Inducible NOS 
(NOS2/iNOS) is expressed by various cells, such as macrophages and fibroblasts, in response to inflammatory stimuli, and it produces large amounts of NO. Since large amounts of $\mathrm{NO}$ are toxic, $\mathrm{NO}$ has been implicated in the pathophysiology of several inflammatory conditions ${ }^{[9-12]}$. In such diseases, under conditions of high and sustained NO production, reactive nitrogen oxide species (RNS) (such as nitrogen dioxide, $\mathrm{NO}_{2}$; dinitrogen trioxide, $\mathrm{N}_{2} \mathrm{O}_{3}$; and peroxynitrite, $\mathrm{ONOO}^{-}$) are formed by reactions with reactive oxygen species (ROS), and may result in nitrosative or oxidative stress ${ }^{[13,14]}$. Some experimental and clinical evidence indicates that pharmacological inhibition of iNOS triggers anti-inflammatory responses, which may be of clinical use $\mathrm{e}^{[12,13]}$. Furthermore, since NO can interact with other inflammatory mediators, it may be part of a system of dual or multiple inhibitors of the inflammatory cascade $^{[14]}$.

Based on these assumptions, the aim of this study was to locally and systemically assess the effect of adjunctive SDD treatment on nitrosative stress in moderate to advanced chronic periodontitis.

\section{Materials and methods Materials}

The following primary monoclonal antibodies were used: anti iNOS (R\&D Systems, RD-MAB9502, MN, USA), anti 3-nitrotyrosine (3NT) (Novus Europe, KA0445, ABNOVA, Cambridge, UK). Mouse IgG1 Negative Control (X0931), and diaminobenzidine (LSAB System-HRP kit K0679) were purchased from Dako (Glostrup, Denmark), and 3NT ELISA Kit (KA0445) was purchased from ABNOVA c/o EMBLEM (Heidelberg, Germany). Sulfanilamide (SULF), N-(1-naphthyl)ethylenediamine dihydrochloride (NEDD), vanadium(III) chloride $\left(\mathrm{VCl}_{3}\right)$, methanol, and diethyl ether were purchased from Merck (Darmstadt, Germany) and Sigma-Aldrich (Taufkirchen, Germany). All chemicals were of analysis grade.

\section{Study population}

The test group ( $n=174)$, consisting of subjects with untreated chronic periodontitis, was recruited from the Department of Periodontology, University of Medicine and Pharmacy Iuliu
Hatieganu, Cluj Napoca, Romania. Their medical and dental histories were obtained, and the patients underwent clinical and radiographic evaluation during a pre-screening visit in order to diagnose periodontal disease $\mathrm{e}^{[15,16]}$. The inclusion criteria were as follows: age, 30-60 years; at least 16 teeth present; at least 3 teeth in the same sextant; diagnosis of moderate to advanced chronic periodontitis; at least 2 sites with probing depths (PD) $\geq 5 \mathrm{~mm}$ together with bleeding on probing (BOP); $\geq 5$-mm clinical attachment level (CAL) loss; and radiographic evidence of alveolar bone height loss. The exclusion criteria included chronic treatment with any medication known to affect periodontal status, such as antibiotics and non-steroidal anti-inflammatory drugs; clinically significant or unstable organic diseases; compromised healing potential, such as with connective tissue disorders or bone metabolic diseases; females who were pregnant or lactating, or who were of childbearing potential and not utilizing birth control or abstinence; documented allergies to tetracycline; cigarette smokers; active infectious diseases; and immunocompromised patients or those taking steroid medications. All dental care related to this trial was provided at no cost to the enrolled participants. Subjects who were eligible based on the clinical pre-screening visit and provided written informed consent were recruited and randomized between May 2008 and May 2011. The last subject completed the clinical trial in July 2011. The study protocol was reviewed and approved by the ethics committee of University of Medicine and Pharmacy "Iuliu Hatieganu," Cluj-Napoca, Romania.

\section{Study design and periodontal clinical measurements}

This was a double-blind, placebo-controlled, randomized, 3-month clinical study with 2 treatment arms: SRP+SDD or $\mathrm{SRP}+$ placebo. The SRP+SDD group received doxycycline capsules (20 mg of doxycycline, twice daily) and the $\mathrm{SRP}+$ placebo group received placebo capsules (filled with cornstarch, twice daily). The study design included 8 visits (Figure 1). Patient eligibility was evaluated during the prescreening visit (visit 1 ). After 1 week, during the screening visit (visit 2), the periodontal status at 6 aspects per tooth (mesio-buccal, mid-buccal, disto-buccal, mesio-lingual, mid-

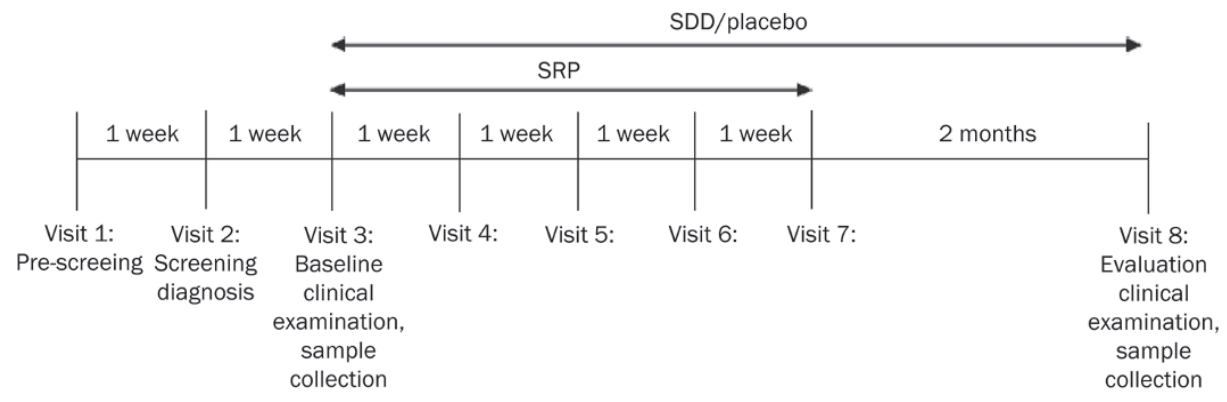

Figure 1. Study design: Visit 1=pre-screening; Visit 2=screening and chronic periodontitis diagnosis; Visit 3=baseline periodontal clinical examination, blood and gingival tissue sample collection, full-mouth scaling of all supragingival and subgingival deposits of plaque and calculus, beginning of SDD or placebo treatment; Visit 4, 5, 6, and 7=root planing and SDD or placebo treatment; Visit 8=evaluation of periodontal clinical indices, blood and gingival tissue sample collection. SRP=scaling and root planing; SDD=subantimicrobial-dose doxycycline. 
lingual, and disto-lingual) was assessed using a standard manual periodontal probe (DB764R, Aesculap AG, Tuttlingen, Germany). At the baseline visit (visit 3), which took place 1 week after the screening, and at the evaluation visit (visit 8), which was performed 3 months after the baseline visit at the time of therapy completion, the clinical periodontal indices $\mathrm{PD}, \mathrm{BOP}$ and CAL were measured.

At baseline, full-mouth scaling of all supragingival and subgingival deposits of plaque and calculus was performed using both hand and ultrasonic instruments, along with local anesthetic, as needed. After the scaling, patients were randomized to the SRP+SDD $(n=87)$ and SRP+placebo $(n=87)$ groups, and coded bottles with corresponding capsules were provided. The patients were instructed to take the capsules twice a day at approximately 12-h intervals, $1 \mathrm{~h}$ before meals. Over the following 4 weeks (visits 4, 5, 6, 7), patients returned for root planing sessions (1 quadrant weekly) performed with local anesthesia, following a clockwise route starting from the upper right and ending at the lower right quadrant.

A single experienced periodontist performed all the clinical measurements and the non-surgical periodontal therapy during the trial. At the baseline and evaluation visits, samples were collected for gingival histological examination and blood tests.

\section{Histopathological and immunohistochemical analysis}

In all patients, tooth extractions were indicated. Thirty-six patients with chronic periodontitis that provided written consent were selected for gingival biopsies; in each group, 6 tooth extractions were performed at baseline, and 12 tooth extractions were performed at the completion of treatment. Immediately after the extraction of the tooth, a piece of gingival tissue was excised using a scalpel. The biopsies were fixed in $10 \%$ phosphate-buffered formalin for $24 \mathrm{~h}$, embedded in paraffin wax, cut into 3-5- $\mu \mathrm{m}$ sections, and stained with hematoxylin and eosin (HE) and Masson's Trichrome (TRI).

A semi-quantitative analysis for edema, fibrosis, necrosis, and dysplasia was performed, with a score of 0-4: 0 (none); 1 (mild); 2 (moderate); 3 (marked); or 4 (severe, diffuse). The inflammatory cell influx, independent for neutrophils and mononuclear cells, was evaluated by counting the number of cells in 20 high-power fields (Cell B basic imaging software, Olympus), and defined as: 0 (0-5 inflammatory cells); 1 (5-10 inflammatory cells); 2 (10-30 inflammatory cells); or 3 (more than 30 inflammatory cells) ${ }^{[12,17-19]}$.

For immunohistochemistry, the sections were incubated with primary monoclonal antibodies against iNOS and 3NT, diluted in $1 \%$ phosphate-buffered saline-bovine serum albu$\min ^{[20]}$. The secondary antibody (labeled streptavidin biotin) was applied, followed by incubation with diaminobenzidine. After washing with distilled water, the slides were counterstained with Mayer hematoxylin for $5 \mathrm{~min}$. Negative controls for each sample were prepared by replacing the primary antibody with mouse IgG1 Negative Control. The number of strongly dense immunoreactive cells was counted in 5 randomly selected microscopic high-power fields (400×) (35.450 $\mu \mathrm{m}^{2}$ each). Semi-quantitative scoring of the stained sections was employed. The proportion of immunopositive cells from the total number of inflammatory cells was expressed as a percentage. Measurements were performed 3 times in representative sections ${ }^{[15,21,22]}$. We used an Olympus BX51 microscope with an Olympus SP 350 digital camera. Cell B basic imaging software (Olympus) was used for the semi-automated counting of infiltrated leukocytes and immunoreactive cells.

\section{Serum nitric oxide evaluation}

Fasting venous blood samples were collected, and serum was separated after centrifugation at $1500 \times g$ for $10 \mathrm{~min}$. The samples were assayed immediately or stored until analysis at $-80^{\circ} \mathrm{C}$ for biochemical tests and at $-20^{\circ} \mathrm{C}$ for enzyme-linked immunosorbent assays (ELISAs).

The Griess reaction was used as an indirect assay to determine the total serum nitrite $\left(\mathrm{NO}_{2}^{-}\right)$and nitrate $\left(\mathrm{NO}_{3}^{-}\right)$as a measure of the degree of $\mathrm{NO}$ production $\left(\mathrm{NO}_{\mathrm{x}}\right)$. Serum samples were passed through 10-kDa filters (Sartorius AG, Goettingen, Germany) and deproteinized by methanol/diethyl ether (3/1, $v / v)$ (sample: methanol/diethyl ether, 1:9, $v / v)^{[22]}$. In brief, 100 $\mu \mathrm{L}$ of $\mathrm{VCl}_{3}(8 \mathrm{mg} / \mathrm{mL})$ was added to $100 \mu \mathrm{L}$ of the supernatant for the reduction of nitrate to nitrite, followed by the addition of the Griess reagents, $50 \mu \mathrm{L}$ of SULF ( $2 \%$ ) and $50 \mu \mathrm{L}$ of NEDD $(0.1 \%)$. After $30 \mathrm{~min}$ of incubation at $37^{\circ} \mathrm{C}$, the absorbance was read at $540 \mathrm{~nm}$. Serum $\mathrm{NO}_{\mathrm{x}}$ was expressed as nitrite $\mu \mathrm{mol} / \mathrm{L}^{[23]}$.

Serum levels of protein-bound 3NT adduct formed by the nitration of tyrosine-containing proteins were estimated using an ELISA Kit. The results were expressed as $3 \mathrm{NT} \mu \mathrm{mol} / \mathrm{L}^{[24]}$.

\section{Statistical analysis}

All results were expressed as the meantstandard deviation (SD) whenever data were normally distributed; otherwise, the median and [Q1; Q3] were reported $(\mathrm{Q} 1=$ first quartile; $\mathrm{Q} 3=$ third quartile). Statistical comparisons between two independent groups were performed using the Student's $t$-test (with equal and unequal variances according to the results of the F-test) whenever data were normally distributed; otherwise, a non-parametric test was applied. The Student's paired $t$-test was applied to compare baseline values with follow-up values for normally distributed data. Statistical comparisons between the two treatment groups were performed using ANOVA tests. Pearson's and Spearman's correlation analyses were used to calculate statistical relationships between parameters. Values of $P<0.05$ were considered to be statistically significant. Analyses were performed using SPSS (Statistical Package for the Social Sciences) 16.0 for Windows (SPSS Inc, USA).

\section{Results}

There were no significant differences in age or sex between the two treatment groups (Table 1). Subjects within the groups were generally well matched. All 174 patients that enrolled for the study completed the trial. No complications or infections were observed throughout the study period. 
Table 1. Demographic characteristics of the patients.

\begin{tabular}{|c|c|c|c|c|}
\hline \multirow{2}{*}{ Parameter } & \multicolumn{2}{|c|}{$\mathrm{SRP}+$ placebo } & \multicolumn{2}{|c|}{ SRP+SDD } \\
\hline & Baseline & 3 months & Baseline & 3 months \\
\hline$n(174)$ & 87 & 87 & 87 & 87 \\
\hline \multicolumn{5}{|l|}{ Age (years) } \\
\hline Mean & 43.12 & 43.12 & 41.5 & 41.5 \\
\hline Average & $30-60$ & $30-60$ & $30-60$ & $30-60$ \\
\hline Gender (M/F) & $43 / 44$ & $43 / 44$ & $42 / 45$ & $42 / 45$ \\
\hline
\end{tabular}

$\mathrm{SRP}=$ scaling and root planning; SDD=subantimicrobial-dose doxycycline.

\section{Periodontal clinical measurements}

Significantly smaller values of PD, BOP, and CAL were observed at 3 months compared to baseline in both investigated groups (Table 2). At baseline, there were no differences in the periodontal clinical indices between the two groups. At 3 months, only the PD $(P=0.002)$ and BOP $(P=1.15 \mathrm{E}-07)$ scores were smaller in the SRP+SDD group than in SRP+placebo group.

\section{Histopathological and immunohistochemical analysis}

Gingival samples stained prior to treatment with HE and TRI presented with an intense inflammatory cell infiltrate, predominantly mononuclear and focally distributed, with many lymphocytes and plasma cells, a discrete presence of macrophages, and the foci of polymorphonuclear leukocytes (PMNs). In addition, edema, fibrosis, and necrosis were found. After 3 months, all SRP+placebo group parameters had smoothly decreased $(P<0.05)$, but in the SRP+SDD group, they were significantly reduced $(P<0.001)$ (Table 3$)$.

The immunohistochemical analysis of iNOS at baseline revealed that inflammatory cells, some epithelial cells, and some fibrocytes were iNOS ${ }^{+}$. PMNs had demonstrated the most intense immunostaining (Figure 2A, Table 4). After 3 months of treatment, both groups showed reduced iNOS $^{+}$ staining, mostly due to the decreased expression of iNOS in the mononuclear leukocytes (Figure 2B, 2C, Table 4). The reduction in iNOS $^{+}$leukocytes was smaller in the SRP+placebo group than in the SRP+SDD group $(P<0.001)$. Based on the analysis of the percentage of total iNOS ${ }^{+}$leukocytes, it was found that SRP+placebo caused no significant reduction in iNOS, while SRP+SDD caused a small decrease (Table 4). After the treatment, there were significant correlations between iNOS ${ }^{+}$staining and clinical indices only in the SRP+SDD group $(r=0.71-0.85)$.

At baseline, positive staining for $3 \mathrm{NT}$ was found in the epithelium, infiltrated leukocytes, and collagen fibers (Figure $2 \mathrm{D}$, Table 4). The inhibitory effect in the SRP+SDD group was better than that in the SRP+placebo group $(P<0.01)$. The SRP+placebo group showed reduced 3NT staining, mostly in the mononuclear leukocytes (Figure 2E, Table 4), while in the SRP+SDD group, it was reduced in all types of leukocytes

Table 2. Periodontal clinical indices parameters of the patients.

\begin{tabular}{|c|c|c|c|c|c|c|}
\hline \multirow[b]{2}{*}{ Parameter } & \multicolumn{3}{|c|}{$\mathrm{SRP}+$ placebo } & \multicolumn{3}{|c|}{ SRP+SDD } \\
\hline & $\begin{array}{c}\text { Baseline } \\
\text { mean } \pm S D\end{array}$ & $\begin{array}{l}3 \text { month } \\
\text { mean } \pm S D\end{array}$ & $P$ & $\begin{array}{l}\text { Baseline } \\
\text { mean } \pm S D\end{array}$ & $\begin{array}{l}3 \text { month } \\
\text { mean } \pm S D\end{array}$ & $P$ \\
\hline $\mathrm{PD}(\mathrm{mm})$ & $6.18 \pm 0.22$ & $3.42 \pm 0.37$ & $9.06 \mathrm{E}-13$ & $5.90 \pm 0.16$ & $3.25 \pm 0.42$ & 8.26E-12 \\
\hline BOP (\%) & $66.00 \pm 2.50$ & $44.60 \pm 3.10$ & 5.41E-07 & $65.4 \pm 1.02$ & $23.70 \pm 1.12$ & $6.24 \mathrm{E}-10$ \\
\hline $\mathrm{CAL}(\mathrm{mm})$ & $5.69 \pm 0.50$ & $4.1 \pm 0.81$ & 0.001 & $5.80 \pm 1.50$ & $3.90 \pm 1.20$ & $1.93 \mathrm{E}-06$ \\
\hline
\end{tabular}

$\mathrm{PD}=$ probing depths; $\mathrm{BOP}=$ bleeding on probing; $\mathrm{CAL}=$ clinical attachment level; $\mathrm{SRP}=$ scaling and root planning; $\mathrm{SDD}=$ subantimicrobial-dose doxycycline; $P=$ statistical significance baseline compared with 3 months.

Table 3. Periodontal semi-quantitative analysis for inflammatory cell influx, edema, fibrosis and necrosis in gingivomucosal tissue of the patients.

\begin{tabular}{|c|c|c|c|c|c|c|}
\hline \multirow[b]{2}{*}{ Parameter } & \multicolumn{3}{|c|}{ SRP+placebo } & \multicolumn{3}{|c|}{$\mathrm{SRP}+\mathrm{SDD}$} \\
\hline & $\begin{array}{l}\text { Baseline } \\
\text { mean } \pm S D\end{array}$ & $\begin{array}{l}3 \text { month } \\
\text { mean } \pm S D\end{array}$ & $P$ & $\begin{array}{l}\text { Baseline } \\
\text { mean } \pm S D\end{array}$ & $\begin{array}{l}3 \text { month } \\
\text { mean } \pm S D\end{array}$ & $P$ \\
\hline PMN & $3 \pm 0.10$ & $2.09 \pm 0.05$ & 4.31E-13 & $2.90 \pm 0.08$ & $1.81 \pm 0.04$ & 2.39E-29 \\
\hline Monocytes & $2.09 \pm 0.07$ & $1.97 \pm 0.04$ & 0.0482 & $2.09 \pm 0.09$ & $1.81 \pm 0.07$ & 0.0045 \\
\hline Eosinophils & $0.81 \pm 0.04$ & $0.63 \pm 0.05$ & 0.0432 & $0.81 \pm 0.04$ & $0.63 \pm 0.05$ & 0.0553 \\
\hline Necrosis & $3.98 \pm 0.07$ & $3.88 \pm 0.08$ & 0.0324 & $3.89 \pm 0.06$ & $3.82 \pm 0.04$ & 0.0038 \\
\hline
\end{tabular}

$\mathrm{PMN}=$ polymorphonuclear leukocytes; $\mathrm{SRP}=$ scaling and root planning; $\mathrm{SDD}=$ subantimicrobial-dose doxycycline; $P=$ statistical significance baseline compared with 3 months. 


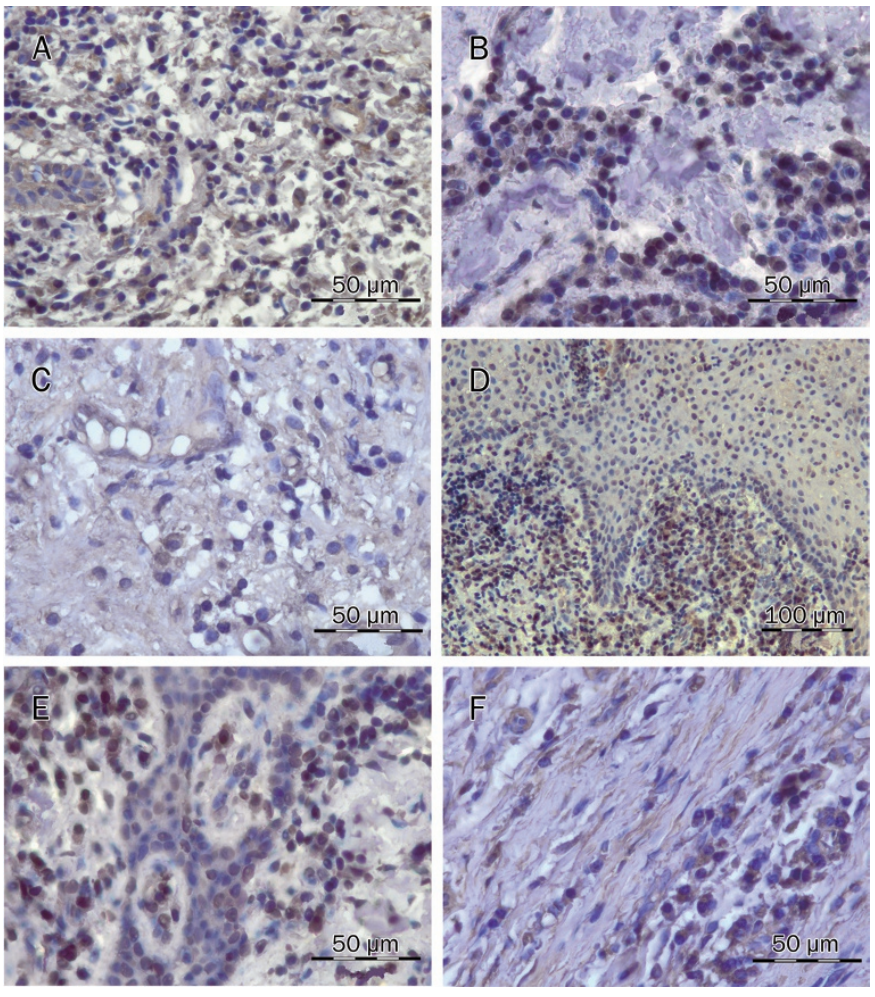

Figure 2. Photomicrographs of representative iNOS-positive and 3-nitrotyrosine-positive stains in gingival tissue from patients with chronic periodontitis (immunohistochemical staining): (A) countless iNOS ${ }^{+} \mathrm{PMNs}$ and monocytes (scale bar, $50 \mu \mathrm{m}$ ); (B) decreased iNOS ${ }^{+}$cells in the $\mathrm{SRP}+$ placebo group (scale bar, $50 \mu \mathrm{m}$ ); (C) sparse iNOS ${ }^{+}$cells in SRP+SDD group (scale bar, $50 \mu \mathrm{m}$ ); (D) countless $3 \mathrm{NT}^{+} \mathrm{PMNs}$ and monocytes (scale bar, $100 \mu \mathrm{m}$ ); (E) decreased $3 \mathrm{NT}^{+}$cells in the SRP+placebo group (scale bar, $50 \mu \mathrm{m}$ ); (F) sparse $3 \mathrm{NT}^{+}$cells in the SRP+SDD group (scale bar, $50 \mu \mathrm{m})$. This figure is representative of 3 measurements $(\times 400)$. iNOS ${ }^{+}=$induced nitric oxide synthase positive cells; $3 \mathrm{NT}^{+}=3$-nitrotyrosine positive cells; $\mathrm{SRP}=$ scaling and root planing; $\mathrm{SDD}=$ subantimicrobial dose of doxycycline.
(Figure 2F, Table 4). The percentage of total $3 \mathrm{NT}^{+}$leukocytes did not significantly decrease in the SRP+placebo group, but in the SRP+SDD group, there was a mild reduction (Table 4). In both the SRP+placebo $(r=0.54-0.77)$ and SRP+SDD $(r=0.99)$ groups, 3NT immunostaining was correlated with iNOS expression. A positive correlation between 3NT immunostaining and the reduction of clinical indices $(r=0.65-0.89)$ was also observed.

\section{Serum nitric oxide evaluation}

After 3 months of treatment, serum $\mathrm{NO}_{x}$ levels registered a small decrease in the SRP+placebo group and a more significant reduction in the SRP+SDD group $(P<0.01)$ (Table 4$)$. Serum $\mathrm{NO}_{x}$ levels were correlated with the clinical periodontal indices $(r=0.58-0.91)$ and the immunohistological staining of iNOS and 3NT $(r=0.59-0.86)$.

Low serum values of $3 \mathrm{NT}$ were definitive after 3 months of treatment in both groups, with a more significant inhibitory effect in the SRP+SDD group than in the SRP+placebo group $(P<0.001)$ (Table 4$)$. There was a satisfactory correlation of serum 3NT with the clinical indices $(r=0.65-0.94)$, tissue iNOS and 3NT $(r=0.61-0.82)$.

\section{Discussion}

In this interventional study, we analyzed nitrosative stress in patients with moderate to advanced chronic periodontal disease that were treated with SRP+SDD or SRP+placebo. The basic supposition was that nitrosative stress damages the periodontal tissue and alveolar bone and may induce systemic inflammatory responses. The magnitude of these consequences depends on the body's defense mechanisms against free radicals. The study was performed in an attempt to systemically and locally assess the efficacy of SRP+SDD in reducing nitrosative stress in moderate to advanced chronic periodontitis.

Table 4. Periodontal immunohystochemical and serum nitrosative stress parameters of the patients.

\begin{tabular}{|c|c|c|c|c|c|c|}
\hline Parameter & $\begin{array}{l}\text { Baseline } \\
\text { mean } \pm S D\end{array}$ & $\begin{array}{l}\mathrm{SRP}+\text { placebo } \\
3 \text { month } \\
\text { mean } \pm \mathrm{SD}\end{array}$ & $P$ & $\begin{array}{l}\text { Baseline } \\
\text { mean } \pm S D\end{array}$ & $\begin{array}{l}\text { SRP+SDD } \\
3 \text { month } \\
\text { mean } \pm S D\end{array}$ & $P$ \\
\hline iNOS $^{+}$Leukocytes & $265.8 \pm 14$ & $181.9 \pm 11.7$ & $1.05 \mathrm{E}-93$ & $265.9 \pm 1.29$ & $92.8 \pm 21.4$ & $1.1 \mathrm{E}-123$ \\
\hline $\mathrm{iNOS}^{+} \mathrm{PMN}$ & $172.3 \pm 19.9$ & $133.3 \pm 34.5$ & $1.58 \mathrm{E}-16$ & $173.02 \pm 2.09$ & $79.3 \pm 29.1$ & $9.67 \mathrm{E}-88$ \\
\hline iNOS $^{+}$Monocytes & $121.3 \pm 31.7$ & $22.8 \pm 3.0$ & 5.7E-68 & $122.6 \pm 2.77$ & $13.5 \pm 7.7$ & $9.67 \mathrm{E}-88$ \\
\hline$\%$ iNOS $^{+}$Leukocytes & $94.8 \pm 3.4$ & $90.75 \pm 5.75$ & 0.06 & $93.9 \pm 0.36$ & $81.9 \pm 10.4$ & 0.05 \\
\hline $3 \mathrm{NT}^{+}$Leukocytes & $219.5 \pm 16.50$ & $137.3 \pm 20.05$ & $1.07 \mathrm{E}-27$ & $217.7 \pm 4.54$ & $101.9 \pm 12.73$ & $3.44 \mathrm{E}-70$ \\
\hline $3 \mathrm{NT}^{+} \mathrm{PMN}$ & $129.9 \pm 13.90$ & $93.3 \pm 12.75$ & 0.0087 & $128.3 \pm 3.85$ & $71.3 \pm 9.62$ & $1.85 \mathrm{E}-32$ \\
\hline $3 \mathrm{NT}^{+}$Monocytes & $89.7 \pm 8.07$ & $44.1 \pm 3.14$ & 5.35E-15 & $91.5 \pm 3.31$ & $30.6 \pm 6.89$ & 7.3E-72 \\
\hline$\% 3 \mathrm{NT}^{+}$Leukocytes & $92.3 \pm 1.12$ & $87.7 \pm 1.21$ & 0.06 & $90.7 \pm 0.94$ & $82.9 \pm 8.98$ & 0.05 \\
\hline $\mathrm{NO}_{x}(\mu \mathrm{mol} / \mathrm{L})$ & $69.89 \pm 2.43$ & $58.69 \pm 3.01$ & 0.0416 & $68.74 \pm 7.31$ & $47.16 \pm 3.10$ & $4.85 \mathrm{E}-24$ \\
\hline 3NT ( $\mu \mathrm{mol} / \mathrm{L})$ & $465.57 \pm 18.63$ & $199.00 \pm 10.29$ & 3.69E-43 & $464.93 \pm 5.76$ & $81.43 \pm 15.86$ & 4.86E-57 \\
\hline
\end{tabular}

iNOS ${ }^{+}=$induced nitric oxide synthase positive cells; $3 \mathrm{NT}^{+}=3$-nitrotyrosine positive cells; $\mathrm{PMN}=$ polymorphonuclear leukocytes; $\mathrm{NO}_{\mathrm{x}}=$ serum nitrites and nitrates; $3 N T=$ serum 3-nitrotyrosine; $\mathrm{SRP}=$ scaling and root planning; SDD=subantimicrobial-dose doxycycline; $P=$ statistical significance baseline compared with 3 months. 
In contrast to supragingival plaque, which is repeatedly destroyed by mastication and/or toothbrushing, subgingival plaque is protected from mechanical removal. Consequently, the subgingival plaque can be considered to be a persistent infection that activates host immuno-inflammatory responses. Currently, most patients with moderate to advanced periodontitis can be treated with nonsurgical therapies for plaque removal ${ }^{[25]}$. In addition, several host-modulatory drugs have been developed to control the immune and inflammatory pathways that are responsible for the breakdown of periodontal tissue. Tetracyclines have pleiotropic therapeutic effects that are mediated by extracellular (ie, inhibition of MMPs) and cellular (ie, inhibition of cytokines, iNOS, and PLA2) inhibitory mechanisms or by their pro-anabolic effect (ie, stimulation of collagen production and osteoblast activity). These mechanisms are unrelated to the antibiotic activity of these drugs $^{[6,7]}$. In the last few decades, many clinical studies have described the beneficial long-term effects of SDD adjunctive to SRP on the basis of the significant reduction in periodontal indices that is observed with this treatment ${ }^{[7,26,27]}$. SRP+SDD therapy also reduced the levels of biomarkers of inflammation and the local destruction of the collagen and bone in periodontal pockets ${ }^{[28,29]}$, gingival crevicular fluid ${ }^{[6,7,30]}$, and saliva ${ }^{[31,32]}$. The optimal duration of SRP+SDD therapy is unknown and probably varies from one patient to another. Other studies have indicated that $90 \%$ of the results are attained after 3 months. Therefore, patients selected for this study were treated for 3 months and then were reassessed ${ }^{[25]}$.

At baseline, histopathological analysis of the HE- and TRIstained slides for the cellular composition of the gingival tissue revealed the already described ongoing inflammatory changes associated with periodontitis ${ }^{[12,14,21]}$. The use of $\mathrm{SRP}+$ placebo and SRP+SDD caused a significant reduction in cellular infiltrate, edema, fibrosis, and necrosis, demonstrating an important anti-inflammatory effect.

$\mathrm{NO}$ is an important inflammatory mediator with both direct and indirect effects. Direct effects include the chemical reactions in which NO reacts directly with its biological target. The indirect effects are mediated by RNS derived from NO metabolism. The direct effects are very rapid reactions that occur at low NO concentrations, and they generally involve heme proteins, such as guanylate cyclase, cytochrome P450, and hemoglobin. The indirect effects require $\mathrm{NO}$ to be activated by superoxide $\left(\mathrm{O}_{2}^{-}\right)$or oxygen to form RNS, which undergo further reactions with their respective biological targets. The indirect effects can be further subdivided into nitrosative and oxidative stress. Nitrosative stress occurs when intermediates are produced from nitrosated thiol, hydroxy, and amine groups ${ }^{[33]}$. The localization and effects of nitrosative stress may be assessed by an analysis of nitrosative damage biomarkers isolated from tissues and biological fluids.

Consistent with the literature, extensive iNOS expression was noted within infiltrating cells at sites of chronic periodontitis. We also observed that resident cells, like epithelial cells and fibrocytes, were intensely iNOS ${ }^{+}$. The role of $\mathrm{NO}$ in modulating PMN infiltration may vary with the type of tissue and the presence of inflammatory disease, but it is thought that low levels of NO reduce inflammatory cell adhesion to the endothelium, and high levels of NO stimulate it. Like other studies, our findings at baseline confirm that high levels of NO induced by iNOS stimulate leukocyte accumulation within the periodontal tissue and reduce clinical periodontal indices ${ }^{[14,19,33]}$. Consequently, in both groups, iNOS reduction diminished inflammatory cell infiltrate, and these were correlated with changes in the periodontal clinical parameters.

The toxicity of $\mathrm{NO}$ is enhanced by the formation of peroxynitrite $\left(\mathrm{ONOO}^{-}\right)$. Peroxynitrite originates from the reaction between $\mathrm{NO}$ and superoxide $\left(\mathrm{O}_{2}^{-}\right)^{[33]}$. The compound itself or its secondary metabolites can cause tyrosine nitration in proteins, producing a $3 \mathrm{NT}$ footprint that is detectable in vivo. Nitrated proteins have been identified in some inflammatory diseases, coronary artery disease, chronic renal failure, rheumatoid arthritis, diabetes mellitus, and cystic fibrosis ${ }^{[34]}$. There is evidence that a number of less abundant proteins (ie, a1-proteinase inhibitor, cytochrome P450, chemokines, manganese superoxide dismutase, and cyclooxygenase) and peptides have altered functions after protein nitration, which may work as negative feedback in the inflammatory process ${ }^{[20]}$. However, basal low-level 3NT has been detected under physiological conditions in most tissues and plasma. That is why complete inhibition of 3NT may not be desirable, as this compound possesses physiological functions, such as the processing of antiinflammatory cytokines and chemokines, which are needed for host defense. Basal protein nitration in tissue is most likely due to $\mathrm{CNOS}$ activity, because iNOS inhibition does not reduce the basal levels of nitrated protein ${ }^{[34,35]}$. In the study population, the positive correlation between iNOS and 3NT staining in the periodontal tissue at baseline proved that this effect was not attributed to physiological nitration, but rather, it was due to increased iNOS expression. Consequently, it may be an indication of increased nitrosative stress ${ }^{[20]}$. The significant changes in periodontal iNOS and 3NT suggest that these tests may be useful for the evaluation of the severity of periodontal damage.

Periodontal bacterial products trigger host immune and inflammatory responses, activating pro-inflammatory mediators. These molecules travel via the blood to other organs and tissues, and this may influence their function and activate a systemic inflammatory response. Numerous lines of evidence support links between the formation of NO-derived oxidants and the development of systemic diseases. Hereby, a very important issue in periodontology is whether periodontal blood markers may predict systemic consequences. Most commonly, RNS have been tracked by measuring some stable metabolites, such as nitrates and nitrites ${ }^{[36]}$. Previously, increased $\mathrm{NO}_{x}$ levels in the saliva and gingival crevicular fluid were found ${ }^{[1,21,36]}$. The subjects treated with SRP+SDD showed a highly significant reduction in serum $\mathrm{NO}_{x}$ and $3 \mathrm{NT}$ levels. More importantly, these results were correlated with improved tissue levels of iNOS and 3NT, along with the periodontal clinical indices. Consequently, serum NO synthesis markers may reflect periodontal NO release and can be used 
as systemic nitrosative stress markers.

The limitations of this study are related to the relatively small number of tissue biopsies (12/group) that were collected and analyzed.

In conclusion, adjunctive SDD host-modulatory therapy with SRP in moderate to advanced chronic periodontitis may be useful against local and systemic inflammatory responses by reducing the generation of nitrosative stress. The ability of SDD to downregulate local and systemic nitrosative stress in vivo seems to be linked to its inhibitory effect on iNOS. Nitrosative stress reduction was correlated with improvements in periodontal clinical indices. These results prove the utility of systemic nitrosative markers in chronic periodontitis diagnosis and prognosis.

\section{Acknowledgements}

This work was financially supported by the Ministry of Education, Research and Youth, CNCSIS grant PNII-IDEI 1273/2008. We would like to thank Synevo Romania SRL for providing the blood samples and Mihaela BANCIU for editing the text.

\section{Author contribution}

Alina Elena PÂRVU designed the study, performed the biochemical tests, and wrote the paper; Sandu Florin ALB conducted patient selection, clinical evaluation and treatment; Alexandra CRĂCIUN performed the ELISA tests; and Marian Aurel TAULESCU performed the histological and immunohistochemical tests.

\section{References}

1 Kirkwood KL, Cirelli JA, Rogers JE, Giannobile WV. Novel host response therapeutic approaches to treat periodontal diseases. Periodontol 2000 2007; 43: 294-315.

2 American Academy of Periodontology. Comprehensive periodontal therapy: a statement by the American Academy of Periodontology. J Periodontol 2011; 82: 943-9.

3 Inaba $\mathrm{H}$, Amano A. Roles of oral bacteria in cardiovascular diseases - from molecular mechanisms to clinical cases: Implication of periodontal diseases in development of systemic diseases. J Pharmacol Sci 2010; 113: 103-9.

4 Bascones-Martínez A, Muñoz-Corcuera M, Noronha S, Mota P, Bascones-llundain C, Campo-Trapero J. Host defence mechanisms against bacterial aggression in periodontal disease: Basic mechanisms. Med Oral Patol Oral Cir Bucal 2009; 14: e680-5.

5 Ababneh KT, Taha AH, Abbadi MS, Karasneh JA, Khader YS. The association of aggressive and chronic periodontitis with systemic manifestations and dental anomalies in a Jordanian population: a case control study. Head Face Med 2010; 6: 30-7.

6 Kumar SS, Mittal M, Khanna P. Role of antibiotics in the treatment of periodontal disease-an overview. Int J Microbiol 2012. DOI: 10.5580/2af7.

7 Golub LM, Greenwald RA. Clinical applications of non-antibacterial tetracyclines. Part II. Pharmacol Res 2011; 64: 549-50.

8 Sgolastra F, Petrucci A, Gatto R, Giannoni M, Monaco A. Longterm efficacy of subantimicrobial-dose doxycycline as an adjunctive treatment to scaling and root planing: a systematic review and metaanalysis. J Periodontol 2011; 82: 1570-81.

9 Menaka KB, Ramesh A, Thomas B, Suchetha Kumari N. Estimation of nitric oxide as an inflammatory marker in periodontitis. J Indian Soc Periodontol 2009; 13: 75-8.

10 Reher VG, Zenóbio EG, Costa FO, Reher P, Soares RV. Nitric oxide levels in saliva increase with severity of chronic periodontitis. J Oral Sci 2007; 49: 271-6.

11 Simão AN, Lozovoy MA, Simão TN, Venturini D, Barbosa DS, Dichi JB, et al. Immunological and biochemical parameters of patients with metabolic syndrome and the participation of oxidative and nitroactive stress. Braz J Med Biol Res 2011; 44: 707-12.

12 Lohinai Z, Mabley JG, Fehér E, Marton A, Komjáti K, Szabó C. Role of the activation of the nuclear enzyme poly(ADP-ribose) polymerase in the pathogenesis of periodontitis. J Dent Res 2003; 82: 987-92.

13 Uğar-Cankal D, Ozmeric N. A multifaceted molecule, nitric oxide in oral and periodontal diseases. Clin Chim Acta 2006; 366: 90-100.

14 Lohinai Z, Benedek P, Fehér E, Györfi A, Rosivall L, Fazekas A, et al. Protective effects of mercaptoethylguanidine, a selective inhibitor of inducible nitric oxide synthase, in ligature-induced periodontitis in the rat. Br J Pharmacol 1998; 123: 353-60.

15 Wu YM, Chen LL, Yan J, Zhuang CY, Gu ZY. Effect of Porphyromonas gingivalis PrtC on cytokine expression in ECV304 endothelial cells and its level in subgingival plaques from patients with chronic periodontitis. Acta Pharmacol Sin 2007; 28: 1015-23.

16 Savage A, Eaton KA, Moles DR, Needleman I. A systematic review of definitions of periodontitis and methods that have been used to identify this disease. J Clin Periodontol 2009; 36: 458-67.

17 Di Nardo Di Maio F, Lohinai Z, D’Arcangelo C, De Fazio PE, Speranza L, De Lutiis MA, et al. Nitric oxide synthase in healthy and inflamed human dental pulp. J Dent Res 2004; 83: 312-6.

18 Watarai $\mathrm{H}$, Warita $\mathrm{H}$, Soma K. Effect of nitric oxide on the recovery of the hypofunctional periodontal ligament. J Dent Res 2004; 83: 338-42.

19 Bezerra MM, Brito GA, Ribeiro RA, Rocha FA. Low-dose doxycycline prevents inflammatory bone resorption in rats. Braz J Med Biol Res 2002; 35: 613-6.

20 Tian J, Yan Z, Wu Y, Zhang SL, Wang K, Ma XR, et al. Inhibition of iNOS protects endothelial-dependent vasodilation in aged rats. Acta Pharmacol Sin 2010; 31: 1324-8.

21 Di Paola R, Mazzon E, Maiere D, Zito D, Britti D, De Majo M, et al. Rosiglitazone reduces the evolution of experimental periodontitis in the rat. J Dent Res 2006; 85: 156-61.

22 Lohinai Z, Stachlewitz R, Virag L, Szekely AD, Hasko G, Szabo C. Evidence for reactive nitrogen species formation in the gingivomucosal tissue. J Dent Res 2001; 80: 470-5.

23 Ghasemi A, Hedayati M, Biabani H. Protein precipitation methods evaluated for determination of serum nitric oxide end products by the Griess assay. J Med Sci Res 2007; 2: 29-32.

24 Miranda KM, Espey MG, Wink DA. A rapid, simple spectrophotometric method for simultaneous detection of nitrate and nitrite. Nitric Oxide 2001; 5: 62-71.

25 Khan J, Brennand DM, Bradley N, Gao B, Bruckdorfer R, Jacobs M. 3-Nitrotyrosine in the proteins of human plasma determined by an ELISA method. Biochem J 1998; 330: 795-801.

26 Greenstein G. Efficacy of subantimicrobial-dose doxycycline in the treatment of periodontal diseases: a critical evaluation. Int $J$ Periodontics Restorative Dent 2004; 24: 528-43.

27 Payne JB, Golub LM, Stoner JA, Lee HM, Reinhardt RA, Sorsa T, et al. The effect of subantimicrobial-dose-doxycycline periodontal therapy on serum biomarkers of systemic inflammation: a randomized, double-masked, placebo-controlled clinical trial. J Am Dent Assoc 2011; 142: 262-73.

28 Lee JY, Lee YM, Shin SY, Seol YJ, Ku Y, Rhyu IC, et al. Effect of 
subantimicrobial dose doxycycline as an effective adjunct to scaling and root planing. J Periodontol 2004; 75: 1500-8.

29 Gu Y, Lee HM, Sorsa T, Salminen A, Ryan ME, Slepian MJ, et al. Nonantibacterial tetracyclines modulate mediators of periodontitis and atherosclerotic cardiovascular disease: A mechanistic link between local and systemic inflammation. Pharmacol Res 2011; 64: 573-9.

$30 \mathrm{Gu}$ Y, Lee HM, Sorsa T, Simon SR, Golub LM. Doxycycline inhibits mononuclear cell-mediated connective tissue breakdown. FEMS Immunol Med Microbiol 2010; 58: 218-25.

31 Emingil G, Gürkan A, Atilla G, Kantarci A. Subantimicrobial-dose doxycycline and cytokine-chemokine levels in gingival crevicular fluid. J Periodontol 2011; 82: 452-61.

32 Preshaw PM, Novak MJ, Mellonig J, Magnusson I, Polson A, Giannobile $W V$, et al. Modified-release subantimicrobial dose doxycycline enhances scaling and root planing in subjects with periodontal disease. J Periodontol 2008; 79: 440-52.

33 Caton J, Ryan ME. Clinical studies on the management of periodontal diseases utilizing subantimicrobial dose doxycycline (SDD). Pharmacol Res 2011; 63: 114-20.

34 Wink DA, Miranda KM, Espey MG. Cytotoxicity related to oxidative and nitrosative stress by nitric oxide. Exp Biol Med (Maywood) 2001; 226: 621-3.

35 Wink DA, Miranda KM, Espey MG, Pluta RM, Hewett SJ, Colton C, et al. Mechanisms of the antioxidant effects of nitric oxide. Antioxid Redox Signal 2001; 3: 203-13.

36 Reher VG, Zenóbio EG, Costa FO, Reher P, Soares RV. Nitric oxide levels in saliva increase with severity of chronic periodontitis. J Oral Sci 2007; 49: 271-6. 